\title{
Star-forming complexes and the spiral structure of our Galaxy $\star, \star \star$
}

\begin{abstract}
D. Russeil ${ }^{\star \star \star}$
Observatoire de Marseille, 2 Place Le Verrier, 13248 Marseille Cedex 04, France

Received 26 April 2000 / Accepted 1 October 2002

Abstract. We have carried out a multiwavelength study of the plane of our Galaxy in order to establish a star-forming-complex catalogue which is as complete as possible. Features observed include $\mathrm{H} \alpha, \mathrm{H} 109 \alpha$, CO, the radio continuum and absorption lines. For each complex we have determined the position, the systemic velocity, the kinematic distance and, when possible, the stellar distance and the corresponding uncertainties. All of these parameters were determined as homogeneously as possible, in particular all the stellar distances have been (re)calculated with the same calibration and the kinematic distances with the same mean Galactic rotation curve. Through the complexes with stellar distance determination, a rotation curve has been fitted. It is in good agreement with the one of Brand \& Blitz (1993). We also investigated the residual velocities relative to the circular rotation model. We find that departures exist over large areas of the arms, with different values from one arm to another. From our data and in good agreement with previous studies, the Galactic warp is observed. It does not seem correlated with the departures from circular rotation. Finally, as segment-like features are noted from the complexes' distribution, we tried to find if they are indicative of a larger underlying structure. Then, we attempted to interpret the complexes' distribution in terms of spiral structure by fitting models with two, three and four logarithmic spiral arms. The four-arm model seems more appropriate to represent the grand design of our Galaxy. In this model the Norma arm and the external arm appear as being the two extremities of a single arm called the Norma-Cygnus arm. The new data and fitted model confirm the four-segment model of Georgelin \& Georgelin (1976), clarifying the arms' design and extension and doubling their known length.
\end{abstract}

Key words. ISM: HII regions - Galaxy: kinematics and dynamics - Galaxy: structure

\section{Introduction}

The determination of external galaxy morphology is classically based on its visual appearance in photographic images (e.g. Hubble 1926). At these wavelengths the young stellar population and their associated HII regions are prominent, and appear preferentially along spiral arms. We propose, in this article, to delineate the spiral structure of our Galaxy as it would be seen in such optical image. As star formation regions are associated with HII regions, the $\mathrm{H} \alpha$ emission is a very good tracer of star-forming complexes and hence of the present spiral structure of galaxies (e.g. Hodge \& Kennicutt 1983; Considère \& Athanassoula 1982,1988). As is observed in external galaxies we do not expect to find a continuous and regular structure, but our goal is to inspect the general trend of the spiral structure of our Galaxy.

\footnotetext{
* Based on observations collected at the European Southern Observatory

$\star \star$ Tables 1 and 3 (table1.ps and table3.txt) are available in electronic form at the CDS via anonymous ftp to cdsarc.u-strasbg. fr $(130.79 .128 .5)$

or via

http://cdsweb.u-strasbg.fr/cgi-bin/qcat?J/A+A/397/133

$\star \star \star$ e-mail: delphine.russeil@oamp.fr
}

Note that the morphology of spiral structure changes when a galaxy is observed in the infrared (Bertin \& Lin 1996). These changes reflect the differing dynamical behaviour of the old and young disk. But the old stellar population seen in the $K$ band for external galaxies varies little between Hubble type, contrary to the optical morphology (Seigar \& James 1998). We will not further discuss this aspect; we focus this article on the morphology of our Galaxy traced by the young objects only.

For external galaxies the distribution of star-forming regions along the spiral arms is generally evident from direct imaging, but for our Galaxy the spiral arms are strung out along the line of sight, leading to the superposition and mixing of information from the different complexes in the spiral arms, making it difficult to distinguish them. Thus in order to study the large scale structure of our Galaxy, we need to identify and catalogue star-forming complexes (molecular clouds, $\mathrm{HII}$ regions and $\mathrm{OB}$ stars) and then determine their distances and hence their spatial distribution. Such a distance determination is based on the identification of the exciting stars of the HII regions belonging to the complexes and/or on the determination of the systemic velocity. In the first case, the stellar distance is determined from the usual spectrophotometric measurements (e.g. Crampton \& Georgelin 1975); in the second case, the systemic velocity is converted into a kinematic distance using a mean Galactic rotation curve. In practice it is rare 
to be able to identify the exciting stars for regions farther than about $6 \mathrm{kpc}$ due to interstellar absorption (e.g. Russeil 1998; Georgelin \& Georgelin 1971; Forbes 1988). However only a few HII regions have known stellar distances. Hence, for a large fraction of HII regions, the only alternative is to have velocity information. Although radio observations are useful to determine velocity, they do not permit a resolution of the distance ambiguity that arises for regions inside the solar circle. In this case $\mathrm{H} \alpha$ information is essential to choose between the far and near distance proposed by the rotation Galactic model. In 1970, Bok et al. successfully applied this method to the tracing of the Carina arm from HII regions and their exciting stars and, in 1976, this study was extended by Georgelin \& Georgelin to the entire Galaxy. They were the first to show that our Galaxy probably has four spiral arms. To do this, they measured the radial velocities of the $\mathrm{H} \alpha$ line for $268 \mathrm{HII}$ regions (using a nontunable Fabry-Perot interferometer) and determined the stellar distances of about 360 exciting stars. But this instrument allowed access only to the brightest HII regions and gave spectral information restricted only to a few percent of the observed field. Also, the acquisition system and data reduction process gave a barycentric velocity of the $\mathrm{H} \alpha$ profiles that was biased by the presence of nightsky lines and other more diffuse nebular contributions.

The brightest HII regions are very good tracers of the spiral structure but it is important to reach fainter and farther HII regions and to follow the diffuse emission in order to refine the identification, the extension and the distance of the starforming complexes. Since then a new instrument has been developed by the Marseille Observatory and has been used to undertake a southern Galactic plane survey (le Coarer et al. 1992). Its main characteristic is to use a scanning Fabry-Perot interferometer which allows us to obtain the spectral information continuously all over the observed field, and to separate the nebular components and the nightsky lines.

In this framework, we have carried out a multiwavelength study ( $\mathrm{H} \alpha, \mathrm{CO}$, radio continuum, exciting stars) of the plane of our Galaxy in order to establish a catalogue, as complete as possible, of star-forming complexes. From this catalogue, the distribution of the complexes is directly available, allowing us to probe the kinematics and the structure of our Galaxy.

In this paper, we present this study. In Sect. 2 we present how we have established the catalogue. In Sect. 3 we discuss the kinematics of our Galaxy (rotation curve and circular rotation departures). In the last section we apply all these aspects to the study of the spiral structure of our Galaxy and discuss the revised model we propose.

\section{The star-forming complexes of our Galaxy}

\subsection{Identification of the complexes}

To investigate the spiral structure and kinematics of our Galaxy, three different approaches can be followed. One can either work directly on individual regions (e.g. Avedisova 1996), or apply a subsequent binning (e.g. Brand \& Blitz 1993), or physically group the regions into star-forming complexes (e.g. Georgelin \& Georgelin 1976). We have applied the method of grouping the different young sources in complexes. Indeed, this allows us to eliminate the "noise" due to spatial and kinematic dispersion of these sources, allowing us to better constrain the systemic velocity and stellar distance and to decrease any spatial and velocity scattering and mixing.

In the first step we define what we call a star-forming complex. As it is well established that the massive young stars and their associated HII regions are born and evolve from giant molecular clouds (e.g. Zuckerman \& Palmer 1974; Burton 1976), we can naturally expect that the different HII regions belonging to the same complex are spatially and kinematically grouped around the parental molecular cloud. Thus a starforming complex can be seen as the grouping of several ionized and molecular sources, their grouping being based on kinematic and spatial considerations. In practice, a complex can be constituted by:

(1) HII regions and ionized patches;

(2) HII region(s), ionized patches and molecular cloud(s);

(3) Molecular clouds alone.

In the last case, the cloud is not actually a massive star-forming complex (it may contain star-forming sites with no HII region yet detectable); its contribution to the spiral structure tracing will be negligible because, as we will see later on, no excitation parameter can be determined.

The grouping of sources of the fourth Galactic quadrant is based on the Fabry-Perot $\mathrm{H} \alpha$ survey of Marseille Observatory started in 1991 at La Silla (Chile). This instrument gives kinematical information continuously in the surveyed regions and, via profile decomposition, provides the separation of the different $\mathrm{H} \alpha$ emissions encountered along the line of sight. Thus it allows the detection of the $\mathrm{H} \alpha$ emission of the discrete HII regions as well as the widely distributed diffuse one. The knowledge of the kinematics of the diffuse emission is very important for linking HII regions together and with molecular clouds; furthermore, in several directions this emission is the only tracer of the spiral arms. The coverage of the $\mathrm{H} \alpha$ survey, the detailed study of the different regions surveyed as well as the instrument description can be found in Amram et al. (1991), le Coarer et al. (1992), Marcelin et al. (1995), Russeil (1998) and Georgelin et al. (2000) and references therein. These $\mathrm{H} \alpha$ data have been supplemented by observations of the CO lines carried out at the SEST radiotelescope (Russeil \& Castets 2002) and a thorough literature search to collect the more complete information about velocities and exciting star parameters.

Concerning the first three Galactic quadrants, we have carried out a similar method using bibliographic data only. The main bibliographic sources used are: Reifenstein et al. (1970), Wilson (1972), Georgelin (1975), Downes et al. (1980), Blitz et al. (1982), Dame \& Thaddeus (1985), Brand (1986), Dame et al. (1986), Jacq et al. (1988), Fich et al. (1990) and Sodroski (1991). We had no access to information on the diffuse emission and faint structures essential to link the different structures together, as the new instrument has made available for the fourth Galactic quadrant. Therefore several small sources have been left isolated, although they could be linked to a complex, because of the lack of information about their environment.

The details of the radial velocities and grouping of sources for the four quadrants are given in Table 1 (Col. 1: complex name, Cols. 2-3: $\mathrm{H} \alpha$ and associated radio sources, 
Cols. 4-8: $\mathrm{H} \alpha$, radio recombination, $\mathrm{CO}$, absorption $\mathrm{H}_{2} \mathrm{CO}$ and $\mathrm{OH}$ velocities, Col. 9: $\mathrm{HI}$ distance indication and Col. 10: notes).

\subsection{The stellar distances}

For each optical HII region we have collected from the literature (mainly via the CDS database) the exciting stars (spectral type between $\mathrm{O}$ and B3) for which spectrophotometric (spectral type, UBV and $\mathrm{H} \beta$ photometry) measurements are given. In general we have averaged the photometric data given by the CDS excluding the photographic ones (considered too inaccurate). In addition, in the directions of the fourth Galactic quadrant we carried out a study of the hot field stars using them to constrain the distance of the diffuse ionized gas layers we have detected. The stellar distance is determined via the usual method. When the spectral type is unknown we have determined it from UBV and $\mathrm{H} \beta$ data or, when the $\mathrm{H} \beta$ line is also unknown, we have assumed that the star is on the main sequence. In this last case, the luminosity and the deduced distance may be underestimated. The visual absorption Av (assuming $R=3.2$ ) was calculated from UBV photometry using the Schmidt-Kaler (1983) calibration and the dereddening relation for the hot stars. The adopted $\mathrm{Mv}$-spectral type calibration is a compilation of the calibrations of Humphreys \& Mc. Elroy (1984, hereafter: HM), Schmidt-Kaler (1983, hereafter: SK) and Vacca et al. (1996, hereafter: VGS). Each of these calibrations covers the HR diagram differently. Then, we carried out a comparison of these calibrations and extracted a mean calibration which allows coverage of the spectral type range from $\mathrm{O} 3$ to $\mathrm{B} 3$ for all luminosity classes with a continuous variation of the absolute magnitude. Table 2 summarizes the adopted calibration. In cases of overlapping, we have taken the averaged absolute magnitude. Such values have been selected without aiming at accuracy; the attempt was to get homogeneously calculated distances. But several sources of uncertainty affect the absolute magnitude determination: first, a systematic error come from the Mv-spectral type calibration itself as any calibration is liable to uncertainty. But we can expect that this uncertainty is probably minimised here because of the using of a compilation of calibrations. Secondly, as Mv-spectral type calibrations are scaled on the distance of the Hyades cluster any change on this distance will systematically affect the distance determination. Perryman et al. (1998) found a distance modulus of 3.33 from Hipparcos, but here we used the old Hyades distance (distance modulus: 3.28 in Schmidt-Kaler 1983) to be able to compare with other results, especially the cluster distances compiled from the literature. Last, any misclassification of a star will induce an error in its $\mathrm{Mv}$ determination. Such uncertainty is typically $0.3 \mathrm{mag}$ ( $\sim 15 \%$ uncertainty on the distance) but can reach 0.7 mag ( $\sim 32 \%$ uncertainty on the distance) for main sequence B stars. But, for stars with only photometric data, this uncertainty can be largely underestimated. We take into account only this uncertainty. Then we estimated that the distance uncertainty is in the range $20 \%-30 \%$.

Note that when the exciting source is composed of a few stars only, we have calculated the distance of each star to
Table 2. The adopted Mv-spectral type calibration.

\begin{tabular}{lccccccc}
\hline \hline & V & IV & III & II & Ib & Iab & Ia \\
\hline O3 & -5.65 & & -6.09 & & & -6.4 & -6.8 \\
O4 & -5.52 & & -5.98 & & & -6.41 & -6.8 \\
O5 & -5.41 & & -5.88 & & & -6.43 & -6.8 \\
O6 & -5.05 & & -5.78 & & & -6.44 & -6.8 \\
O6.5 & -5.0 & & -5.72 & & & -6.45 & -6.8 \\
O7 & -4.94 & & -5.67 & -5.9 & -6.3 & -6.46 & -6.8 \\
O7.5 & -4.88 & & -5.62 & -5.9 & -6.27 & -6.47 & -6.8 \\
O8 & -4.73 & -4.9 & -5.57 & -5.9 & -6.25 & -6.48 & -6.8 \\
O8.5 & -4.52 & -4.9 & -5.52 & -5.87 & -6.25 & -6.48 & -6.8 \\
O9 & -4.46 & -4.9 & -5.46 & -5.85 & -6.25 & -6.49 & -6.8 \\
O9.5 & -4.2 & -4.9 & -5.35 & -5.8 & -6.07 & -6.50 & -6.85 \\
B0 & -3.9 & -4.5 & -5.1 & -5.75 & -6.05 & & -6.9 \\
B0.5 & -3.7 & -4.5 & -4.7 & -5.32 & -5.97 & & -6.9 \\
B1 & -3.2 & -3.8 & -4.4 & -5.25 & -5.8 & & -6.9 \\
B1.5 & -2.81 & -3.3 & -4.02 & -5.05 & -5.77 & & -6.9 \\
B2 & -2.47 & -3.1 & -3.8 & -4.8 & -5.75 & & -6.9 \\
B2.5 & -2.0 & -3.1 & -3.47 & -4.75 & -5.7 & & -6.9 \\
B3 & -1.6 & & -3.0 & -4.7 & -5.65 & & -6.9 \\
\hline
\end{tabular}

determine a mean distance and uncertainty. As the absolute magnitude is the main source of uncertainty, we estimated the uncertainty of the stellar distance of a complex adopting $30 \%$ uncertainty and weighting by $1 / \sqrt{n}$ (with $n$ being the number of stars involved in the distance calculation).

For clusters, we have adopted the mean distance given in the literature, the most often established from the ZAMS fitting method. As the clusters usually have no distance uncertainty, we have selected a sample of open clusters from the CDS (catalogues: Lynga 1987; Ruprecht et al. 1983; Loktin et al. 1994) to estimate it statistically. For each cluster we have determined the dispersion of the different distance determination and used it to estimate the error of the distance. We found no obvious correlation between distance and uncertainty except an increase of the dispersion for clusters farther than $1 \mathrm{kpc}$. Hence we arbitrary split the sample into clusters closer and farther than $1 \mathrm{kpc}$ and calculate the standard deviation: respectively of $0.04 \mathrm{kpc}$ and $0.25 \mathrm{kpc}$ for clusters closer and farther than $1 \mathrm{kpc}$. These values are in agreement with the few error bar determinations of open cluster given in the literature. To summarise, the distance uncertainty for cluster is taken directly from the literature when it is available; if not, for clusters with multiple distance determination, the adopted uncertainty is the dispersion; finally, for clusters with only one distance determination, we adopted the values above.

Thus we have created an updated and homogeneous set of stellar distances.

\subsection{The kinematic distances}

The radial velocity of the complexes is often the only information available for determining the distance. The kinematic distance is calculated by the usual method (e.g. Brand \& Blitz 1993, hereafter BB), which assumes that the complexes are in circular orbits around the Galactic centre, and requires knowledge of the Galactic rotation curve. The hypothesis of circular 
rotation allows us to link the angular velocity to the Vlsr, and the Galactocentric distance to the heliocentric distance. Note that, this last relation being non-linear, the kinematic distance is not uniquely determined for objects inside the solar circle: two heliocentric distances are possible for a given Galactocentric distance.

For objects inside the solar circle, we have calculated the kinematic distance using the rotation curve of Brand \& Blitz (1993). We show in Sect. 3.1 that the rotation curve obtained with our data is in agreement with Brand \& Blitz's curve, hence our choice. For objects outside the solar circle we have adopted a flat rotation curve with velocity $220 \mathrm{~km} \mathrm{~s}^{-1}$. Indeed, our knowledge of the rotation curve outside of the solar circle is mainly based on anticentre objects. This is not necessarily representative of the azimuthally-smoothed outer solar circle rotation curve. Moreover, other spiral galaxies as luminous as the Galaxy are observed to have a flat rotation curve (Bosma 1981; Rubin et al. 1985; Persic et al. 1996).

Several parameters enter into the different steps of the kinematic distance calculation; any change of these parameters will modify the distance determination.

Among these parameters are the components of the solar motion toward the apex, which enter into the calculation of the radial velocity with respect to the local standard of rest. We have adopted the following values: $U_{0}=-10.4 \mathrm{~km} \mathrm{~s}^{-1}$, $V_{0}=14.8 \mathrm{~km} \mathrm{~s}^{-1}$ and $W_{0}=7.3 \mathrm{~km} \mathrm{~s}^{-1}$. These values are those commonly used and their use facilitates comparison with previous velocity data. However, several authors find somewhat different values for different types of objects (Dehnen \& Binney 1998; Chen et al. 1997; Sabas 1997; Pont et al. 1994; Wilson et al. 1991; Crampton \& Georgelin 1975; Balona \& Feast 1974), giving a range of values corresponding to a velocity uncertainty up to $5 \mathrm{~km} \mathrm{~s}^{-1}$.

The second set of parameters are those of the Galactic rotation of the local standard of rest. We have adopted the values recommended by the IAU in 1985 , which are $R_{0}=8.5 \mathrm{kpc}$ and $\theta_{0}=220 \mathrm{~km} \mathrm{~s}^{-1}$. But the determination of these values depends on the sample and on the kind of objects used. Any change in $\theta_{0}$ will drastically affect the slope of the rotation curve, while a change in $R_{0}$ will mainly affect the radial scaling. Currently, several authors suggest a $\theta_{0}$ around $200 \mathrm{~km} \mathrm{~s}^{-1}$ and an $R_{0}$ less than $8 \mathrm{kpc}$ (Brand \& Blitz 1993; Honma \& Sofue 1996; Reid 1993; Miyamoto et al. 1993; Dambis et al. 1995). Recently, Olling \& Merrifield (1998), from mass model calculations, have concluded that a consistent picture emerges only when considering $R_{0}=7.1 \pm 0.4 \mathrm{kpc}$ and $\theta_{0}=184 \pm 8 \mathrm{~km} \mathrm{~s}^{-1}$. Note that if the distances used to trace the structure were kinematic distances alone, any change of $R_{0}, \theta_{0}$ gives rise to a homothetic change of the morphology. Here we mix kinematic and stellar distances. In this case, any change of $R_{0}, \theta_{0}$ induces a shift of kinematic distances relative to the stellar distances. For example, changing $R_{0}, \theta_{0}$ from $8.5 \mathrm{kpc}, 220 \mathrm{~km} \mathrm{~s}^{-1}$ to $7.1 \mathrm{kpc}, 184 \mathrm{~km} \mathrm{~s}^{-1}$ induces a mean relative distance shift of up to $\sim 1 \mathrm{kpc}$.

The last point we must clarify is the resolution of the distance ambiguity for sources inside the solar circle. The first step is to look at the information given by the absorption lines in the line of sight: if absorption lines are found with velocities greater (in absolute value) than those of the source, then far distance is to be preferred. Similarly, if the complex exhibits an $\mathrm{H} \alpha$ counterpart we will prefer the near distance. Finally, the latitude position gives an additional clue concerning the distance: if the latitude is low the far distance will be preferred. Let us note a final case: several complexes exhibit velocities forbidden by the rotation model; in this case the adopted distance is that of the tangential point corresponding to the highest permitted velocity for a given direction.

We have estimated the systemic velocity uncertainty of each complex to be $\pm 5 \mathrm{~km} \mathrm{~s}^{-1}$. This takes into account only measurement uncertainty and statistics. It does not take into account the localized deviations relatively to the circular rotation model.

\subsection{The excitation parameter}

In external galaxies, the main spiral structure is traced by the most active complexes. Hence to quantify the complexes' activity we have calculated the excitation parameter $U$ (in $\mathrm{pc} \mathrm{cm}^{-2}$ ), which is the most appropriate as it is a direct measure of the ionizing photons produced. This parameter is determined from the radio continuum flux via the relation (Schraml \& Mezger 1969):

$U=4.5526\left[a(v, T)^{-1} v^{0.1} T^{0.35} S_{v} D^{2}\right]$

where $T$ is the temperature (in Kelvins), $S_{v}$ is the radio flux (in Jy) at the frequency $v$ and $D$ the distance (in kpc); $a(v, T)$ as defined by Mezger \& Henderson (1967) is close to 1. For each source we have collected, from the literature, the radio continuum flux, when available. The main bibliographic sources used are Caswell \& Haynes (1987), PMN (Wright et al. 1994; Griffith et al. 1993, 1995) and GB6 (Griffith et al. 1990, 1991; Langston et al. 1990; Bennett et al. 1986) catalogues. The excitation parameter for each complex is then calculated from the excitation parameter of the individual sources composing it. But because a lot of individual sources have no radio continuum measurement, the excitation parameter is often underestimated. Note that for molecular clouds the excitation parameter has no meaning. So the complexes composed by molecular clouds only will have artificially low contributions to the arm design. Fortunately, only a few complexes are concerned by this problem.

The frequency distribution of the excitation parameters of complexes, along with the distribution of their distances from the Sun, allow us to estimate the completeness of our sample: it is complete for complexes brighter than $60 \mathrm{pc} \mathrm{cm}^{-2}$. In external galaxies, HII region catalogs show excitation parameter ranging from 10 to $700 \mathrm{pc} \mathrm{cm}^{-2}$ (Rozas et al. 1999, 2000; Gonzalez Delgado \& Perez 1997), large scale structures being generally traced by the brightest regions (brighter than $70 \mathrm{pc} \mathrm{cm}^{-2}$ ). Hence our sample completeness allows us to undertake the study of the spiral structure.

\subsection{The final sample}

The final sample of the star-forming complexes is given in Table 3. The first column is a simple counter. The two next 
Table 4. The recent rotation curves of our Galaxy.

\begin{tabular}{lll}
\hline \hline Reference & Zone & Objects \\
\hline $\begin{array}{l}\text { Sinha (1978) } \\
\text { Burton \& Gordon (1978) }\end{array}$ & quad. I and IV & HI \\
$\begin{array}{l}\text { Gunn et al. (1979) } \\
\text { Clemens (1985) }\end{array}$ & quad. I & HI, CO \\
& North & CO, HI for $R<R_{0}$ \\
Rohlfs et al. (1986) & North + South & CO, HII for $R>R_{0}$ \\
& & HII for $R>R_{0}$ \\
Fich et al. (1989) & North & HI, CO-HII \\
Alvarez et al. (1990) & quad. IV & CO \\
Merrifield (1992) & Ext. Gal. & HI \\
Brand \& Blitz (1993) & all & HII-CO, HI \\
& & Reflection Neb. \\
\hline
\end{tabular}

columns give the Galactic coordinates. For each complex we have determined the systemic velocity (Col. 4), the kinematic distance (Col. 5) and the stellar distance (Col. 8) and its uncertainty (Col. 9) when possible. The stellar distance is determined via a weighted average of the stellar distances of the constitutive objects. When given between parenthesis, the stellar distance is based on photometric data alone. Concerning systemic velocities, they have been calculated from the individual velocities of the constitutive objects, preferably the molecular velocities. Columns 6 and 7 of Table 3 give the uncertainty of the kinematic distance assuming an error of the systemic velocity of $\pm 5 \mathrm{~km} \mathrm{~s}^{-1}$. Finally, the excitation parameter is listed in Col. 10. In the following the final distance adopted is the stellar distance, when known, otherwise the kinematic distance. Note that for a few complexes we have taken into account the departure from circular rotation (see Sect. 2.3) to correct the kinematic distance.

\section{The kinematics of our Galaxy}

\subsection{The Galactic rotation curve}

The study of the Galactic rotation curve is not recent; it has been determined from observations of various objects (HI, CO, HII). Table 4 summarizes some of the most recent rotation curve determinations. The most complete one is the BB rotation curve which is determined from the combination of optical and radio data for the whole Galaxy. Knowledge of the Galactic rotation curve is essential to determine the kinematic distance. But the choice of a general rotation curve is not obvious because they are often determined from restricted Galactic directions (Table 4), and therefore biased by direction-dependant irregularities. Moreover, the large error bars and scatter (Binney \& Dehnen 1997) make it difficult to identify these irregularities and to determine the general rotation curve.

Our goal here is not to produce a new rotation curve but to characterise the velocity-stellar distance correspondence for our sample in order to scale the distance of complexes without stellar distance determination. We then fit a rotation curve through our sample of star-forming complexes. In this framework we have selected, in the initial sample, the complexes with known velocity and stellar distances. The data within $12^{\circ}$ from the Galactic centre and anti-centre are excluded from this analysis because of the degeneracy of the radial velocity.

We first fitted a polynomial, but such an analytic expression fits all the structures of the rotation curve. These structures are due to local motions and they are not representative of the general azimuthally-smoothed rotation curve we need to calculate the kinematic distance of complexes without stellar distance determination.

Hence we adopt the same analytical expression as BB (the choice of this function and its properties are discussed by Fich et al. 1989 and BB 1993):

$\omega / \omega_{0}=a 1\left(R / R_{0}\right)^{a 2-1}+a 3\left(R_{0} / R\right)$

where the independent parameters to optimise are the coefficients $a 1$ and $a 2, R$ and $\omega$ being respectively the Galactocentric distance and the angular rotation velocity. We force the rotation curve to pass through $R_{0}$ and $\theta_{0}$, implying $a 3=1-a 1$. The fit was carried out through the sample (157 complexes) by minimizing the following expression:

$\tau=\frac{1}{N} \sum_{i=1}^{N} \sqrt{\left(\frac{\left(R_{i}-R_{t}\right)^{2}}{\sigma_{R_{i}}{ }^{2}}+\frac{\left(\omega_{i}-\omega_{t}\right)^{2}}{\sigma_{\omega_{i}}{ }^{2}}\right)}$

where $\sigma_{R_{i}}$ and $\sigma_{\omega_{i}}$ are the uncertainties of the $N$ objects of the sample (calculated from the distance and radial velocity uncertainties given Table 3 ) and $R_{\mathrm{t}}$ and $\omega_{\mathrm{t}}$ the coordinates of the theoretical points.

The algorithm used to minimize the $\tau$ is the Minuit package, with the migrad and/or simplex search routines (Nelder $\&$ Mead 1965). Since the fitting code requires starting values and is not able to detect convergence to secondary minima, we have carried out an iterative strategy using the $\tau$ value to guide us toward the best parameter set. Several sets of solution with less than $1 \%$ differences in $\tau$ are found. They give the same flat rotation curve (Fig. 1, plotted with $a 1=0.705$ and $\left.a 2=0.35 \times 10^{-8}\right)$.

We have also applied the fitting routine adopting $R_{0}=$ $7.1 \mathrm{kpc}$ and $\theta_{0}=184 \mathrm{~km} \mathrm{~s}^{-1}$. Once again we find a flat rotation curve.

Our sample, fully based on stellar distance determinations, shows behaviour close to that of the sample of Brand \& Blitz (1993); error bars, for complexes outside the solar circle, are similar. For complexes inside the solar circle one also notes good agreement, but we cannot compare error bars as they obtain such points from a purely kinematic approach: the tangent method which finds, from the maximum velocity observed in each direction, the distance for only one position. This also explains why they have data closer to the Galactic centre.

In conclusion, the rotation curve obtained from our data is very close to the Brand \& Blitz (1993) curve, which confirms our choice to calculate the kinematic distance (Sect. 2.3). The dispersion of the points with respect to the mean rotation curve is quite large; this can be attributed to departures from circular rotation (see next section).

\subsection{Deviations from circular rotation}

For those complexes without known stellar distances, tracing the spiral structure requires that we correct the kinematic 


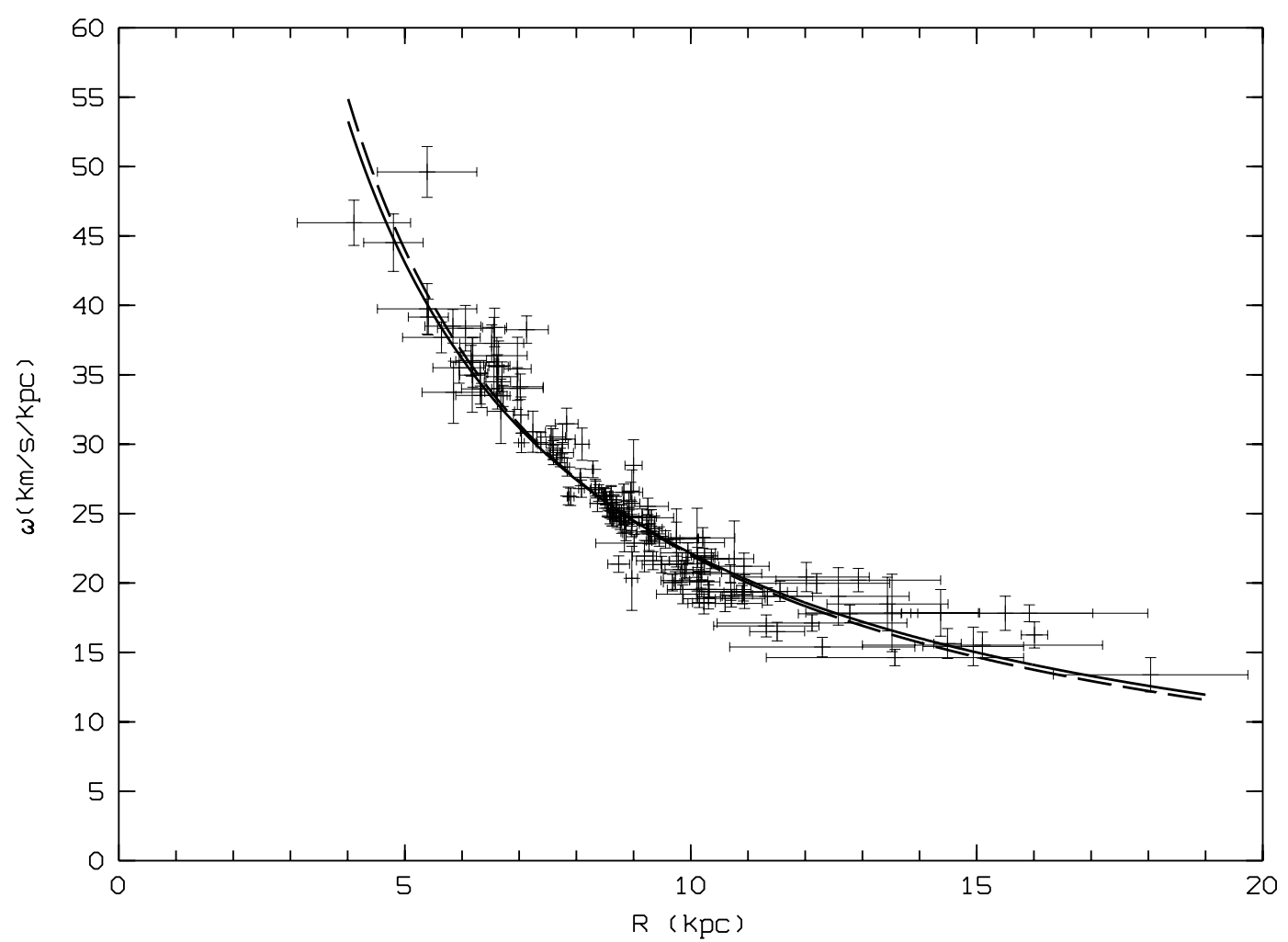

Fig. 1. The computed rotation curve (dashed line) compared with the Brand \& Blitz (1993) curve (solid line).

distances of the complexes within longitude intervals where departures from circular motion have been identified. But do we have to correct the distances of the other complexes? The answer is that we must determine the origin of these departures: if they are due to local phenomenon, the correction must be applied locally, in case of a global origin we will have to apply the correction to every complex in a single arm.

At large scales, the propagation of a spiral density wave in the rotating Galaxy causes variations in the gravitational potential, giving rise to systematic motions of stars and gas in the spiral arms (Roberts 1969; Yuan 1969). At medium scale, velocity departures can be caused by energetic processes happening in the Galactic disk, such as photo-ionisation, stellar winds from massive stars, supernova explosions and high velocity cloud impacts onto the Galactic disk (e.g. Avedisova \& Palous 1989; Heyer \& Terebey 1998). All these processes are sources of compression and acceleration of the interstellar matter, triggering star formation. We can suspect that objects formed in these conditions maintain these accelerations and then exhibit peculiar velocities with respect to the Galactic rotation model. Star formation propagation triggered by $\mathrm{OB}$ associations and supernovae explosions has already been invoked to explain the observed structures in the Magellanic clouds and the galaxy M33 (Rosado et al. 1996; Oey \& Massey 1994). Bubbles, shells and cavities in the interstellar medium observed at different wavelengths are the remnants of such processes induced by stellar evolution. For example, the HI bubbles observed in our Galaxy are the relics of a shock wave expansion in the interstellar medium, induced by supernovae or stellar winds. It is therefore important to identify these large structures and to catalogue the young objects and HII regions they have triggered. This requires a multispectral approach, to detect them and to quantify the velocity departures. Such a study has allowed us to identify and delineate a part of the Galactic plane around $l=290^{\circ}$ exhibiting velocity departures of $7 \mathrm{~km} \mathrm{~s}^{-1}$ (Georgelin et al. 2000).

To quantify the circular rotation departures we calculate, for each complex of the previous sample, the difference between the rotation velocity $\theta$ deduced from the radial velocity and the rotation velocity $\theta_{\text {mod }}$ deduced from the stellar distance. Figure 2 presents the difference $\theta-\theta_{\text {mod }}$ versus the Galactic longitude of complexes belonging to the three closest arms. We note that the three groups exhibit different mean circular rotation departures: Sagittarius-Carina arm $3.3 \pm 14.2 \mathrm{~km} \mathrm{~s}^{-1}$, Perseus arm $-21 \pm 10.3 \mathrm{~km} \mathrm{~s}^{-1}$ and External (also called Cygnus arm) $17.4 \pm 15.0 \mathrm{~km} \mathrm{~s}^{-1}$.

Such velocity departures have already been observed. Velocity anomalies in the Perseus and Carina arms have been known for a long time (Rickard 1968; Humphreys 1970, 1972); more recently Alvarez et al. (1990) have shown molecular velocity excesses of $12 \mathrm{~km} \mathrm{~s}^{-1}$ in the Carina arm and Heyer \& Terebey (1998) show evidence that expanding motions are present within the Perseus arm. These velocity departures were studied in the radial velocity and seemed to be localized. When studying them from the rotation velocity we show that departures exist throughout large parts of the arms. Indeed for the Perseus arm they are present between $90^{\circ}$ and $150^{\circ}$. In these directions the complexes linked to the external arm do not exhibit the same value of deviation, suggesting that these velocity departures are real. The velocity deviation known for the 


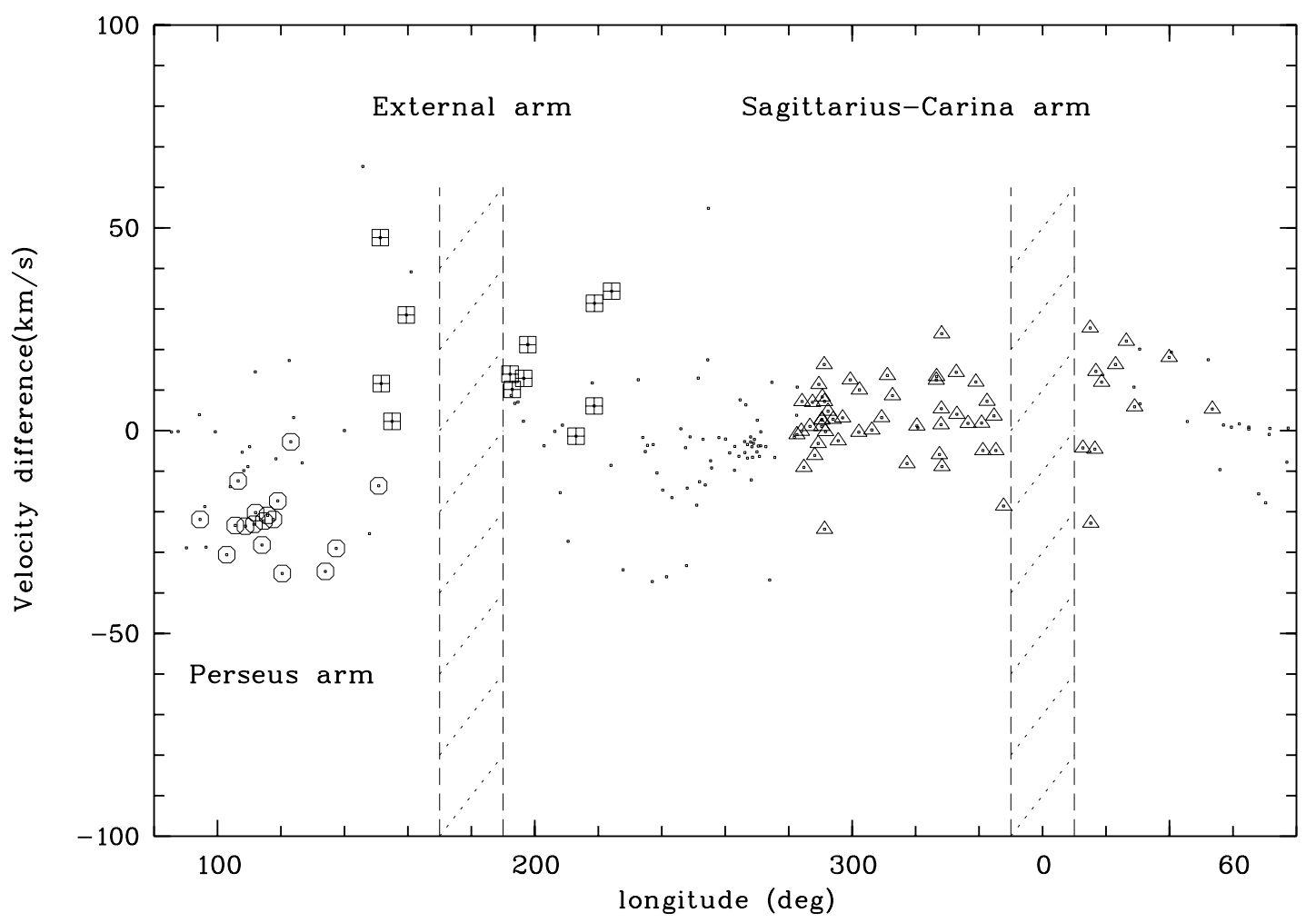

Fig. 2. Velocity difference $\left(\theta-\theta_{\text {mod }}\right)$ versus longitude for each arm: circular, square, triangle and dot symbols correspond respectively to Perseus, External, Sagittarius-Carina and local arms. The two hatched areas have been excluded. They correspond to $12^{\circ}$ from the Galactic centre and anti-centre directions where there is velocity degeneracy. The typical velocity error bar is $\pm 6 \mathrm{~km} \mathrm{~s}^{-1}$.

Carina arm appears to be present all along the arm until $50^{\circ}$. The deviation of the regions of the external arm between $150^{\circ}$ and $220^{\circ}$ is clear. But for this arm such departures must be used with caution because the regions are far away and thus the stellar distance is more difficult to evaluate. Indeed, any systematic distance uncertainty can lead to velocity departures (Sitnik \& Mel'nik 1996).

Thus, at least locally, each arm seems to follow its own rotation curve.

\section{The structure of our Galaxy}

\subsection{The large scale pattern}

Although the spiral nature of our Galaxy has been accepted for a long time, its precise design is still widely discussed. Indeed, neither the number of arms nor their pitch angle are yet fixed. Vallée in 1995 reminded us that the different studies carried out between 1980 and 1995 generally propose a structure with two or four-arms and a pitch angle between $6.5^{\circ}$ and $18^{\circ}$. Recently, Armaral \& Lépine (1997) and Lépine et al. (2001) described the spiral structure by a superposition of two and four-armed wave harmonics and Englmaier \& Gerhard (1999), combining COBE NIR data, HI and molecular gas longitude-velocity diagrams, found a four-arm spiral pattern between the corotation of the Galactic bar $(3.5 \mathrm{kpc})$ and the solar circle.

In parallel, Drimmel (2000) found that emission profiles of the Galaxy in the $K$-band (corresponding mainly to the stellar emission) are consistent with a two-arm structure, whereas the
$240 \mu \mathrm{m}$ emission from dust is compatible with a four-arm structure. Such facts have already been observed for external galaxies: grand design spiral structure can be seen in $K^{\prime}$ while a flocculent appearance is exhibited on blue images. This suggests the stellar and gaseous disks are decoupled (Thornley 1996; Grosbol \& Patsis 1998; Elmegreen et al. 1999).

Moreover one can notice, from many external galaxies, numerous spurs, arm branching, arm segmentation, arches and bridges (Elmegreen 1990; Elmegreen \& Elmegreen 1995; Russell \& Roberts 1993). In fact, the morphological appearance of galaxies depends on how much the local- and intermediate-scale features are important relative to the grand design of the spiral structure: in some galaxies (e.g. M51) local and intermediate scales appear to coexist within a global grand design spiral structure; other galaxies exhibit more dominant local and intermediate scale features as well as more dominant multi-arm structures (e.g. M101) in place of a clear grand design and, finally, in other system only flocculent patterns are apparent (e.g. NGC 2841). However, several morphological properties of external spiral galaxies are commonly observed (Seigar \& James 1998; Block 1982; Kennicutt 1982a; Kennicutt 1981): the azimuthal winding up angle for the strongest arm ranges from $17^{\circ}$ to $310^{\circ}$, the pitch angle from $4.1^{\circ}$ to $15.8^{\circ}$ (Sa galaxies have a mean pitch angle of $6^{\circ}$ whereas the Sc galaxies have a mean pitch angle of $18^{\circ}$ ) and the arm width from 0.4 to $7.5 \mathrm{kpc}$.

Concerning our Galaxy, a number of criteria (extent of the bulge, bulge to disk ratio, etc.) have been used by various 
authors to classify its type between Sb and Sc (Kerr 1993). The other well-established facts for our Galaxy are the following:

- The tangential directions, corresponding to maxima in the thermal radio continuum, $\mathrm{HI}$ and $\mathrm{CO}$ (Bronfman 1992) emission, are: $33^{\circ}, 55^{\circ}, 282^{\circ}, 309^{\circ}, 328^{\circ}$ and $337^{\circ}$. Several of these tangential directions are also found from other kinds of tracers (Ortiz \& Lépine 1993; Bloemen et al. 1990; Hayakawa et al. 1981).

- The Sagittarius and the Carina arms are linked (e.g. Russeil 1997) tracing the well known Sagittarius-Carina arm located at about $2 \mathrm{kpc}$ from the sun (e.g. Georgelin et al. 1994, 1996, 2000).

- The parts of Perseus and Sagittarius arms nearest to the Sun are well delineated, the Sun lying between them (King 1989; Feinstein 1994). It is even located in a spur - the Orion Spur (King 1989).

Figure 3 presents the complexes' distribution projected onto the Galactic plane, with error bars. Complexes with error bars greater than $5 \mathrm{kpc}$ have been removed. Such large error bars are mainly due to the fact that the directions to these complexes viewed from the Sun are tangent to isovelocity contours. Fortunately this concerns only few complexes which, moreover, have low excitation parameters.

Taking into account the error bars, a simple visual inspection of Fig. 3 does not show any large-scale spiral structure. This is not surprising because even for external galaxies, where no distance uncertainty exist, the grand design structure is not always obvious.

In Fig. 3, only the Carina arm (around $x=-6 \mathrm{kpc}, y=$ $6 \mathrm{kpc}$ ), the Perseus arm (around $x=3 \mathrm{kpc}, y=10 \mathrm{kpc}$ ), the local arm and the presence of segments of arms (around $x=-2 \mathrm{kpc}, y=3.5 \mathrm{kpc} ; x=-2 \mathrm{kpc}, y=5.5 \mathrm{kpc}$ and $x \in[0,10] \mathrm{kpc}, y \in[-6,0] \mathrm{kpc})$ are clearly seen. These features suggest us that in the fourth Galactic quadrant we can expect at least 3 arm-like structures, in the first quadrant 2 arm-like structures and in the second quadrant at least the Perseus arm structure. The question now is: are these structures connected to each other?

To investigate if a large-scale pattern exists in our Galaxy, we propose to fit the distribution of the complexes by logarithmic spirals even if this representation is not totally realistic. Spiral arms have previously been approximated by logarithmic spirals in external galaxies (Kennicutt 1982b; Vorontsov-Vel'yaminov 1987; Arp 1964; Danver 1942; Seiden \& Gerola 1979).

In this model, in polar coordinates $(R, \theta)$, the galactocentric distance of each arm is given by: $R(\theta)=r_{0} \mathrm{e}^{-p \theta}$, where $p$ is the pitch angle (the pitch angle at each point of the spiral is the angle between the spiral and the tangent to the centred circle of radius $R$ ) and $r_{0}$ is the initial radius. To take into account possible slight pitch-angle variation along the arm (e.g. Russell \& Roberts 1992) and/or to estimate its stability, we have assumed the following dependence: $p=\sum_{i=0}^{3} a_{i}\left(\frac{r}{r_{0}}\right)^{i}$.
Table 5. The optimized parameters: $\mathrm{r}_{0}, a_{i}, \Sigma,<W>$ and $<\Delta>$ are given in $\mathrm{kpc}, \mathrm{deg}, \mathrm{kpc}, \mathrm{kpc}$ and $\mathrm{kpc}$ respectively.

\begin{tabular}{|c|c|c|c|}
\hline & $\begin{array}{l}\text { two-arm } \\
\text { model }\end{array}$ & $\begin{array}{c}\text { three-arm } \\
\text { model }\end{array}$ & $\begin{array}{c}\text { four-arm } \\
\text { model }\end{array}$ \\
\hline$r_{0}$ & 4.0 & 3.7 & 3.7 \\
\hline$a_{0}$ & 5.2 & 8.2 & 9.1 \\
\hline$a_{1}$ & 0.4 & 0.02 & 0.4 \\
\hline$a_{2}$ & 0.0 & 0.0 & -0.006 \\
\hline$a_{3}$ & $0.2 \times 10^{-3}$ & 0.0 & $-0.6 \times 10^{-4}$ \\
\hline$r_{0}$ & 3.5 & 3.5 & 3.5 \\
\hline$a_{0}$ & 12.4 & 12.1 & 12.1 \\
\hline$a_{1}$ & -2.1 & 0.2 & 0.02 \\
\hline$a_{2}$ & 0.08 & 0.03 & -0.01 \\
\hline$a_{3}$ & 0.0 & -0.05 & -0.002 \\
\hline$r_{0}$ & - & 4.5 & 4.5 \\
\hline$a_{0}$ & - & 13.7 & 12.1 \\
\hline$a_{1}$ & - & -1.6 & 0.06 \\
\hline$a_{2}$ & - & 0.01 & 0.002 \\
\hline$a_{3}$ & - & 0.0 & -0.003 \\
\hline$r_{0}$ & - & - & 4.5 \\
\hline$a_{0}$ & - & - & 11.3 \\
\hline$a_{1}$ & - & - & 0.03 \\
\hline$a_{2}$ & - & - & -0.002 \\
\hline$a_{3}$ & - & - & -0.002 \\
\hline$\Sigma$ & 0.77 & 0.62 & 0.66 \\
\hline$<W>$ & 1.55 & 1.25 & 1.32 \\
\hline$<\Delta>$ & 2.45 & 2.46 & 2.12 \\
\hline
\end{tabular}

The theoretical parameters $a_{i}$ for each arm are optimized by minimizing the following expression:

$\Sigma=\frac{1}{N} \sum_{i=1}^{N} \min \left(U_{i} \sqrt{\frac{\left(x_{i}-x_{t}\right)^{2}}{\sigma_{x_{i}}^{2}}+\frac{\left(y_{i}-y_{t}\right)^{2}}{\sigma_{y_{i}}^{2}}}\right)$

where $x_{t}^{2}+y_{t}^{2}=R^{2}, x_{i}$ and $y_{i}$ are the Cartesian coordinates of the $N$ observed points in the Galactic plane, $\sigma_{x_{i}}$ and $\sigma_{y_{i}}$ are the uncertainties deduced from the distance uncertainty, $U_{i}$ (the excitation parameter) is the weight we assign to each observed point and the function "min" means the selection of the closest theoretical point. The algorithm and strategy used to minimize the value of $\Sigma$ are the same as in Sect. 3.1.

The quality of the fit is then given by the variable $\Sigma$, which represents the mean distance between the observed points and the theoretical model. This is, in fact, a direct evaluation of the mean dispersion of the observed points with respect to the fitted model, hence an estimation of the half width of the arms.

As it is not possible, with the simple model we adopt, to impose all the known constraints on the fit, the strategy we have followed is to fit the models and check if the constraints are satisfied. Thus to delineate the general trend of the Galactic structure we have fitted models with two, three and four spiral arms through the spatial distribution of the complexes. The results of the best fit for each model, the deduced mean arm width $(<W>)$ and the estimated mean arm-to-arm distance $(<\Delta>)$ are given in Table 5 and plotted in Fig. 4. The tangential directions found are given in Table 6.

All the models are displayed in Fig. 4. We must now take into account the constraints in order to choose the best one. 
Table 6. The tangential directions.

\begin{tabular}{ccc}
\hline \hline & Radius $(\mathrm{kpc})$ & Longitude $\left({ }^{\circ}\right)$ \\
\hline two-arm model & 4.0 & 28 \\
& 5.7 & 42 \\
& 6.9 & 306 \\
& 4.8 & 325 \\
\hline three-arm model & 3.7 & 25 \\
& 4.5 & 32 \\
& 7.1 & 56 \\
& 8.4 & 283 \\
& 6.4 & 312 \\
& 4.9 & 324 \\
\hline four-arm model & 3.7 & 25 \\
& 4.6 & 32 \\
& 7.0 & 56 \\
& 8.4 & 284 \\
& 6.6 & 309 \\
& 5.1 & 323 \\
\hline
\end{tabular}

Note: the observed tangential directions (Bronfman 1992) are $33^{\circ}, 55^{\circ}, 282^{\circ}, 309^{\circ}, 328^{\circ}$ and $337^{\circ}$.

As is naturally expected, the arm length of the two-arm model is large $\left(\sim 540^{\circ}\right.$ and $\left.\sim 580^{\circ}\right)$ while the three-arm and the fourarm model arms (respectively $450^{\circ}, 450^{\circ}, 360^{\circ}$ and $230^{\circ}, 270^{\circ}$, $360^{\circ}, 440^{\circ}$ ) are shorter. The shortest arm is obtained for the Scutum-Crux arm $\left(230^{\circ}\right)$ with the four-arm model. The pitch angles and arm widths of the fitted models fall in the typical range of spiral galaxies. The pitch angles exhibit only slight distance dependance; the mean values (at $R_{0}$ ) are $7.9^{\circ}$ (for the two-arm model), $10.8^{\circ}$ (three-arm model) and $11.3^{\circ}$ (four-arm model). The two-arm model gives arms with quite different pitch angles while three-arm and four-arm models exhibit more similar pitch angle from arm to arm.

Observed tangential directions (Bronfman 1992): $33^{\circ}, 55^{\circ}$, $282^{\circ}, 309^{\circ}, 328^{\circ}$ and $337^{\circ}$.

Let us now carry out a comparative analysis quadrant by quadrant. In the following we will refer to the arm name identified in Fig. 5.

The fourth quadrant $\left(l \in\left[270^{\circ}, 360^{\circ}\right]\right)$ :

This quadrant is the most interesting because it is where most of features can be seen. The different fitted models delineate the same structures: the Carina, Crux and Norma arms. But the two-arm model places the Sun in the Carina arm, which implies that the Carina arm tangential direction at $282^{\circ}$ is not accounted for. Moreover it connects the Local Arm to the Carina Arm, which is not observed. Hence these arguments do not favour this model. For three-arm and four-arm models the tangential directions are quite well respected. But the tangential direction expected at $328^{\circ}$ is found at $323^{\circ}$ for the four-arm model and $324^{\circ}$ for the three-arm model. This direction corresponds to a part of the Norma Arm where there is a lack of tracers, implying a weaker constraint to the fit at this position.

From CO longitude-velocity diagrams of the fourth Galactic quadrant Bronfman et al. (2000) show evidence for the presence of four arms. One of them, commonly named the 3 -kpc arm, is seen with a tangential direction $\sim 337^{\circ}$. Whatever our models, this tangential direction at $337^{\circ}$ is not found, but this direction points toward a group of far complexes $(x \sim$ $-5 \mathrm{kpc}, y \sim-3 \mathrm{kpc}$ ) with high excitation parameters, attributed (in the four-arm model) to the beginning of an arm. It is not possible to confirm the coincidence of these far complexes with the $3 \mathrm{kpc}$ arm but the near faint complexes at $(x \sim-2, y \sim+2 \mathrm{kpc}$; Fig. 5 bottom, solid line) certainly belong to it. From Bronfman et al. (2000) one can also note that the Norma Arm appears clearly in FIR star-formation complexes: its tangential position $\left(\sim 328^{\circ}\right)$ is correlated with an FIR enhancement produced by embedded ultracompact HII regions. This can explain why in our work we find a gap of complexes at the Norma arm tangency, as ultracompact HII regions cannot be detected in optical and radio hydrogen line emission. In contrast the Crux arm stands out clearly in molecular emission while the Carina arm, which is a major arm in our complex distribution, appears weaker. One suspects that the higher the excitation parameter the more the parental molecular cloud has been destroyed.

The first quadrant $\left(l \in\left[0^{\circ}, 90^{\circ}\right]\right)$ :

For the two-arm model the tangential directions are not respected, while it is for other models. The Scutum tangent is double in a number of tracers (Table 1 of Englmaier \& Gerhard 1999): in our three-arm and four-arm models this is attributed to the beginnings of two different arms.

Solomon et al. (1987) traced a face-on picture of warm molecular clouds in the first Galactic quadrant $\left(l\right.$ between $8^{\circ}$ and $90^{\circ}$ ), delineating three arm-like structures. Two of them correspond well to the Sagittarius and Perseus arms as delineated by our three- and four-arm models. The third structure they identify is an inner structure they attribute to a central CO annulus. We have no counterpart for this structure except perhaps complexes we attributed to the beginning of the Crux and Norma arms. Kulkarni (1982) carried out an HI survey giving information about spiral arms beyond the solar circle. He identified the Perseus arm from $l=65^{\circ}$ to $150^{\circ}$ and the Cygnus arm between $l=30^{\circ}$ to $110^{\circ}$. From our four-arm model the Perseus arm and the Cygnus arms also are found to cut the Solar circle around $l=30^{\circ}$ and $l=65^{\circ}$ respectively. One can note that in the first quadrant the Cygnus arm is mainly extrapolated by the fitting routine between the Norma arm (fourth quadrant) and the External arm (second quadrant) complexes, as no complexes are found to trace it in this quadrant 1.

The second and third quadrants $\left(l \in\left[90^{\circ}, 270^{\circ}\right]\right)$ :

In the three-arm and four-arm fitted models, the Perseus arm is unambigously delineated. Beyond it one finds the presence of complexes with a very sparse distribution. They are associated with the Cygnus arm even if it is not clear whether these complexes are organized into an arm structure. Previously, Heyer \& Terebey (1998) identified small and compact clouds beyond the Perseus arm and Digel et al. $(1994,1996)$, demonstrated the presence of molecular material within the far outer Galaxy. In parallel, Kaltcheva \& Hilditch (2000), from the distribution of bright $\mathrm{OB}$ stars towards longitudes $245^{\circ}$ and $225^{\circ}$, suspected the presence of an external arm beyond the Perseus arm.

Comparing our models with information in the literature, it appears that the two-arm model does not satisfactorily describe the large-scale structure of our Galaxy, while the threearm and four-arm models give very close solutions with several arguments in favour of the four-arm models. These two 

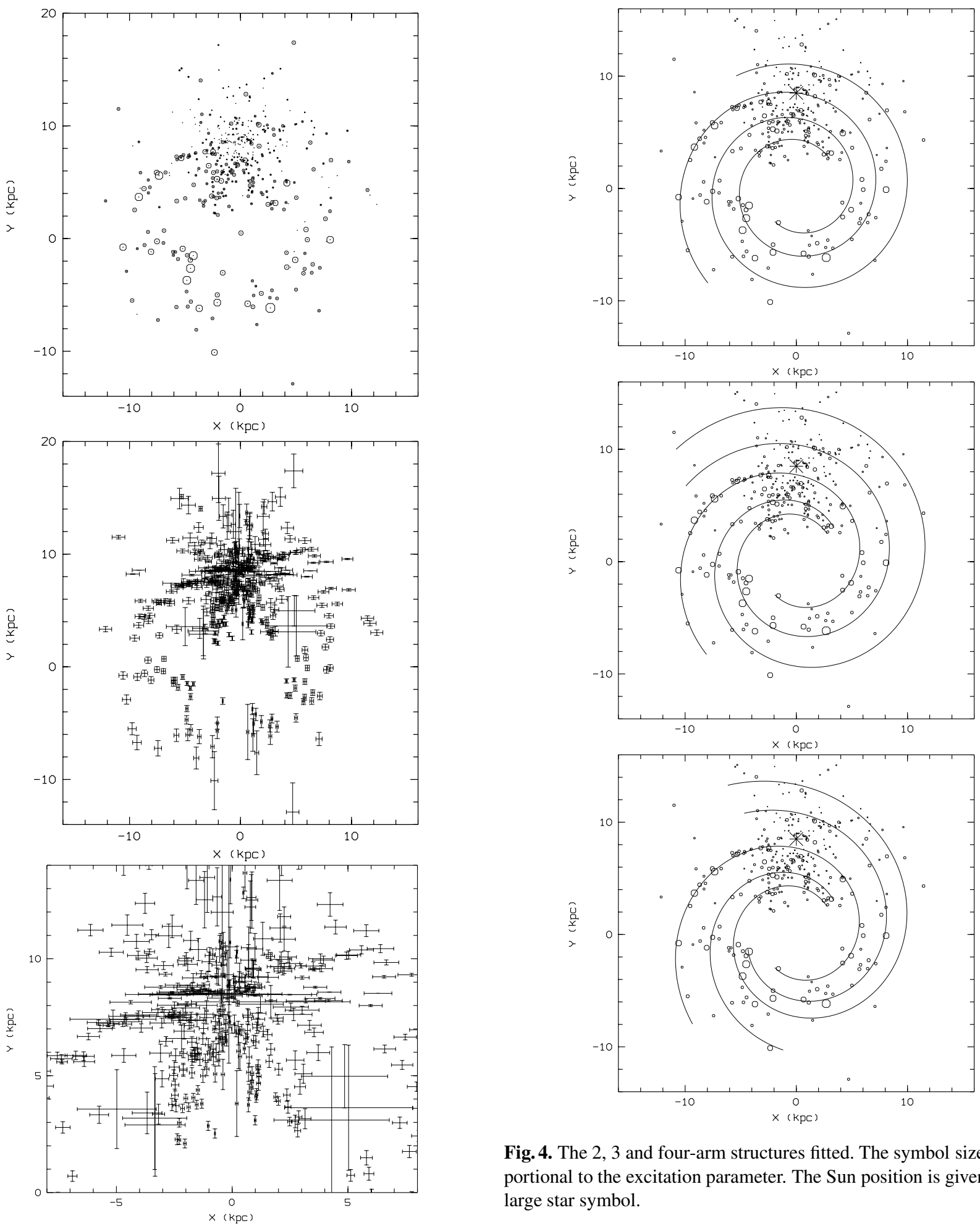

Fig. 4. The 2, 3 and four-arm structures fitted. The symbol size is proportional to the excitation parameter. The Sun position is given by the large star symbol.

Fig. 3. Top: Distribution of complexes. The symbol size is proportional to the excitation parameter. Middle: Same as above, but with only errors bars displayed. Bottom: Enlargement of the middel panel.

models are in agreement with the four-segment model from Georgelin \& Georgelin (1976). The main difference between the three- and four-arm models is that the former connects the Norma segment to the Perseus segment and the Scutum-Crux to the Cygnus parts while the latter connects only the Norma

part to the Cygnus part. This ambiguity is likely due to the lack of complexes in the Norma part (around $X=-5 \mathrm{kpc}$ and $Y=1 \mathrm{kpc}$, Fig. 4) and the possible extension in the first quadrant of the Cygnus arm. The fit quality is, therefore, poorer in these areas.

To test the influence of the solar parameters $\left(R_{0}\right.$ and $\left.\theta_{0}\right)$ on the complexes' distribution we used $R_{0}=7.1 \mathrm{kpc}$ and $\theta_{0}=$ $184 \mathrm{~km} \mathrm{~s}^{-1}$ : this does not change drastically the overall picture. 


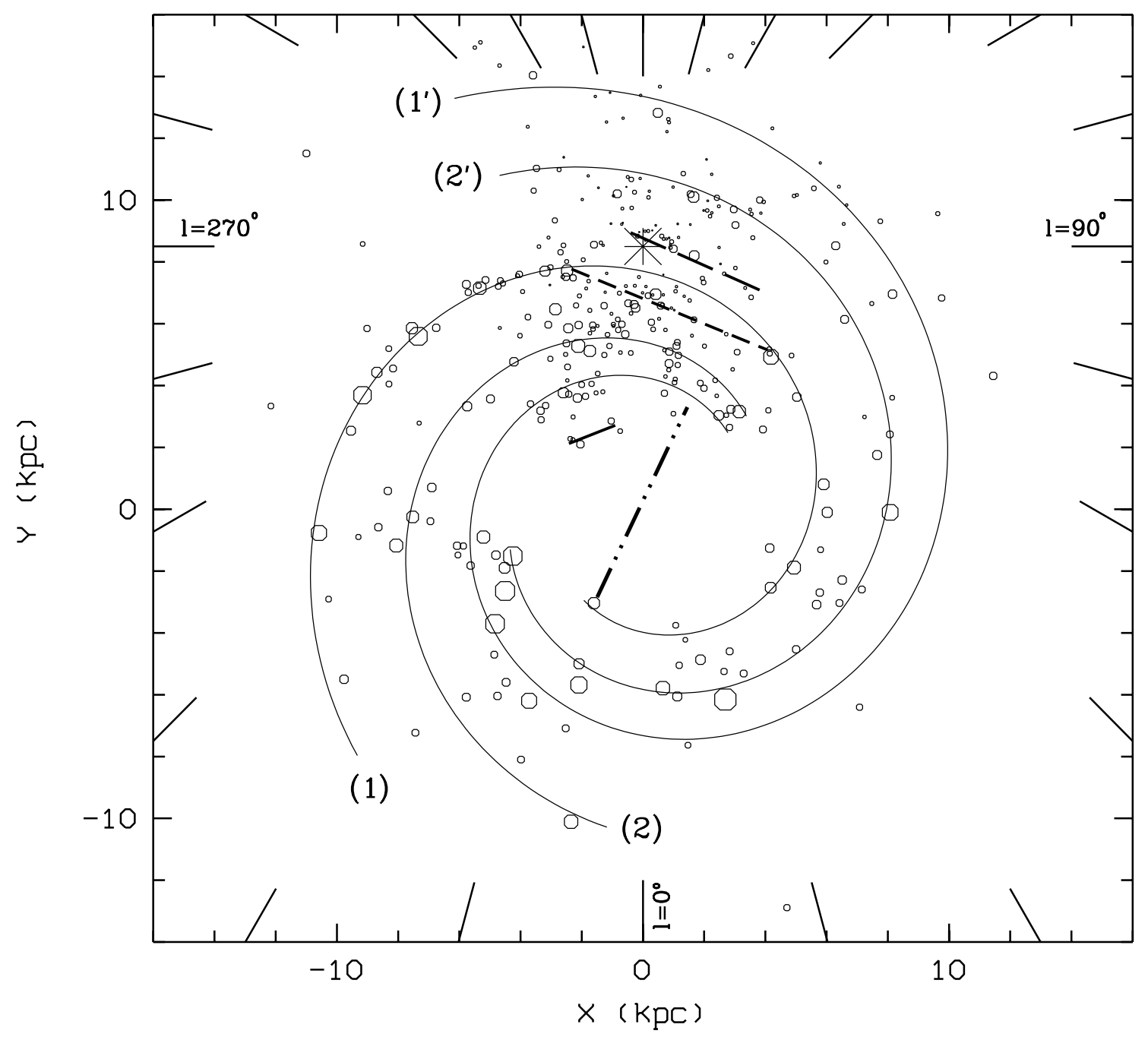

Fig. 5. The adopted four-arm model. The symbol size is proportional to the excitation parameter. The Sun position is given by the large star symbol. All the complexes are plotted. 1: Sagittarius-Carina arm, 2: Scutum-Crux arm, 1': Norma-Cygnus arm and 2': Perseus arm. We have also sketched the local arm feature (long dashed line), the bar orientation and length (dashed-dot-dot line) from Englmaier \& Gerhard (1999), the expected departure from a logarithmic spiral arm observed for the Sagittarius-Carina arm (short dashed line) and finally a feature certainly linked to the three-kpc arm (solid line)

As we already noticed we have fitted regular logarithmic arms, but it is commonly seen on external galaxies that arms do not have a regular design. This fact is illustrated here because a precise study of the nearest parts of the Sagittarius-Carina arm places it at $\sim 2 \mathrm{kpc}$ from the Sun while the fitted spiral passes at $\sim 1 \mathrm{kpc}$ : the axis of the arm must be moved slightly inward in regions just inside the Sun (Fig. 5, dashed line).

The four arm model is consistent with the recent work of Amaral \& Lépine (1997) from a dynamical approach. They show that the spiral structure of our Galaxy can be represented by the superposition of two- and four-arm components with a pitch angle of about $14^{\circ}$; such a solution looks like a pure four-arm structure when the components are in phase. This is another argument in favor of the four-arm model (Fig. 5). Comparing the adopted model to the model of Georgelin \& Georgelin (1976) one can see that the general design is preserved but now the arms are more clearly defined and more extended. The Norma segment appears now to be connected to the Cygnus segment, forming a single extended arm symmetrical to the Sagittarius-Carina arm. The Scutum-Crux arm, which is the shortest, is symmetric with respect to the Perseus arm. The Carina arm appears to be a major arm; sometimes such a major arm is observed in grand design external galaxies. This suggests a grand design structure coexisting with smaller-scale structures, rather than a purely floculent one.

One of these smaller-scale structures is the local arm. Figure 5 (long dashed line) shows a hint of the presence of the local arm: we note a collection of aligned complexes parallel to the Sagittarius arm and including the Sun. Unfortunately, from our data it is impossible to define its characteristics. Jacq et al. (1988) have already showed the existence of a collection of molecular clouds aligned with a pitch angle of $22^{\circ}$ and linking up the local material to the Sagittarius arm.

Three arms out of four start close to the end of the Galactic bar major axis. In barred galaxies, arms are generally connected to the bar (Sellwood \& Sparke 1988). Moreover, for our 


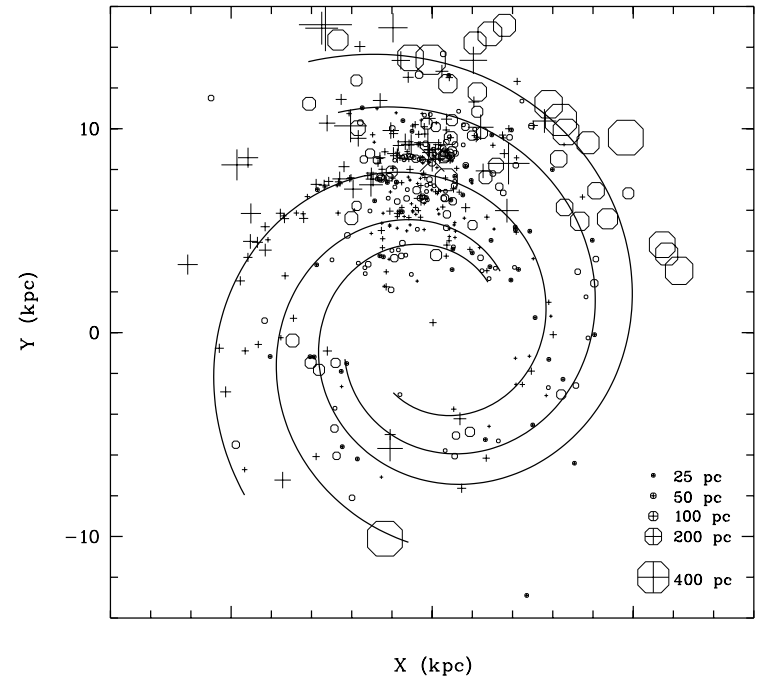

Fig. 6. Representation of the Galactic warp. Circular symbols and crosses correspond respectively to complexes above and below the Galactic plane. The symbol size is proportional to the distance from the plane. For clarity, complexes closer than $1 \mathrm{kpc}$ from the Sun have not been plotted.

Galaxy it has already been suspected that arms start near the bar's ends (e.g. Lòpez-Corredoira et al. 1999; Englemaier \& Gerhard 1999).

\subsection{The Galactic warp}

The Galactic warp was revealed early from HI data: Kerr (1957) showed that the warp starts at the solar circle and reaches a distance of $3 \mathrm{kpc}$ from the Galactic plane at a galactocentric distance of $16 \mathrm{kpc}$, and Burton (1988) has shown that the warp lies above (positive warp) and below (negative warp) the Galactic plane, respectively at $l=90^{\circ}$ and $l=270^{\circ}$, seemingly oriented in the direction of the Magellanic Clouds. More recently the Galactic warp has been observed from young star distributions (Smart et al. 1997), HII regions (Guibert et al. 1978; Fich \& Blitz 1984) and even from stars selected from the IRAS catalogue (Djorgovski \& Sosin 1989). The Galactic warp can also be seen (Fig. 6) from our catalogue of star-forming complexes. In agreement with previous studies we find that the northern part is above the Galactic plane, the southern part being slightly below it. Especially, the Norma-Cygnus arm exhibits a clear positive warp between $l=60^{\circ}$ and $220^{\circ}$. The negative warp of the southern part of our Galaxy is less clear. The Carina arm, which is a major arm of our Galaxy, is mainly located slightly below the Galactic plane. Inversely, the Perseus arm between $l=90^{\circ}$ and $150^{\circ}$, which is well known for velocity departures, exhibits only a slight warp. These two effects seem therefore to be unrelated. We find a mean thickness of the inner Galactic disk of 76 pc. This is in good agreement with the $73 \mathrm{pc}$ and $70 \mathrm{pc}$ found by Bronfman et al. respectively from ultracompact HII regions (Bronfman et al. 2000) and CO data (Bronfman 1992). Also, Bronfman (1992) finds a negative warp in the third and fourth quadrants, as we obtain with our data.

\section{Conclusion}

A new catalogue of star-forming complexes has been established from a multiwavelength study of the Galactic plane. Each complex is a grouping of HII regions, diffuse ionized gas, molecular clouds and OB stars. For each complex the systemic velocity, the kinematic distance, the stellar distance and the excitation parameter have been determined. A rotation curve has been fitted through those complexes for which it has been possible to calculate the stellar distances. Our curve is in good agreement with that of Brand \& Blitz (1993). We also performed a study of velocity departures relative to the circular rotation model. We find that such departures exist in large parts of the arms, with different values from one arm to another. This can explain, at least partly, the quite large dispersion of the complexes around the mean rotation curve. The Galactic warp is also found, in good agreement with previous studies. In addition we note that circular rotation departure and warp do not seem obviously correlated.

Taking into account the error bars, a simple visual inspection of the distribution of the complexes does not show any large-scale spiral structure. The only clear features are the Carina and the Perseus segments and the local arm. These features suggest that we can expect at least three arm-like structures in the fourth Galactic quadrant, two arm-like structures in the first quadrant and at least the Perseus arm structure in the second quadrant. To test if these arm-like structures can underline a grand design structure as observed in external galaxies, we fitted the distribution of all of the Galactic complexes of our sample with two-, three- and fourlogarithmic-arm models of the spiral structure. On the basis of established characteristics for our Galaxy and external galaxies, the two-arm model can be clearly excluded while the three- and four-arm models remain in strong competition - with a slight preference, however, for the latter. In this model the Norma arm and the external arm appear as the two extremities of a unique arm called the Norma-Cygnus arm. The new data and fitted model confirm the four-segment model of Georgelin \& Georgelin (1976) defining the arm design and extension (doubling the known length of the arms). The Carina arm is a major arm, as is sometimes observed in external galaxies, suggesting a grand design structure, coexisting with smaller-scale structures rather than a purely floculent one.

Additional multiwavelength observations will be required to solve the problems which have been encountered. In particular, it appears that ultracompact HII regions, detectable in the FIR, can also be used to trace the actual spiral structure. It would be useful to complete our picture of the Galaxy with such regions. It would be an especially great improvement to detect and measure the velocities and/or distances of such ultracompact HII regions in the northern hemisphere so as to define, in particular, the Cygnus arm design in the first quadrant.

Finally, since many large radio and optical surveys are being collected, the next step will be to compare our data with external spiral galaxies in order to determine the characteristics we have found for our Galaxy: pitch angle variation, 
arm-to-arm distance, arm length and width, arm structure and circular rotation departure.

Acknowledgements. DR thanks Y.M. Georgelin, C. Adami, P. Amram, S. Arzano, J. Boulesteix, J.L. Gach, J. Caplan, Y.P. Georgelin, A. Laval, E. le Coarer, M. Marcelin and A. Viale for reading the manuscript, typing the table, discussions, suggestions, observations and instrument and software maintenance.

\section{References}

Alvarez, M., May, J., \& Bronfman, L. 1990, ApJ, 348, 495

Amaral, L. H., \& Lépine, J. R. D. 1997, MNRAS, 286, 885

Amram, P., Boulesteix, J., Georgelin, Y. M., et al. 1991, The Messenger, 64, 44

Arp, H. 1964, ApJ, 139, 1045

Avedisova, V. S. 1996, Astro. Lett., 22, 443

Avedisova, V. S., \& Palous, J. 1989, Bull. Astron. Inst. Czechosl., 40, 42

Balona, L., \& Feast, M. W. 1974, MNRAS, 167, 621

Bennett, C., Lawrence, C., Burke, B., Hewitt, J., \& Mahoney, J. 1986, ApJS, 61, 1

Bertin, G., \& Lin, C. C. 1996, in Spiral structure in galaxies a density wave theory (Publisher: Cambridge, MA MIT Press), 271

Blitz, L., Fich, M., \& Stark, A. A. 1982, ApJS, 49, 183

Block, D. L. 1982, A\&A, 109, 336

Bloemen, J. G. B., Deul, E. R., \& Thaddeus, R. 1990, A\&A, 233, 437

Bok, B. J., Hine, A. A., \& Miller, E. W. 1970, IAU symp., 38, 246

Bosma, A. 1981, AJ, 86, 1825

Brand, J., \& Blitz, L. 1993, A\&A, 275, 67

Brand, J. 1986, Ph.D. Thesis, Leiden

Bronfman, L. 1992, in The center, bulge and disk of the Milky Way, ed. L. Blitz, p. 131

Bronfman, L., Casassus, S., May, J., \& Nyman, L. 2000, A\&A, 358, 521

Burton, W. B. 1988, in Lecture notes in physics, 306, 94

Burton, W. B., \& Gordon, M. A. 1978, A\&A, 63, 7

Burton, W. B. 1976, ARA\&A, 14, 275

Caswell, J. L., \& Haynes, R. F. 1987, A\&A, 171, 261

Chen, R., Asiain, R., Figueras, F., \& Torra, J. 1997, A\&A, 318, 29

Clemens, D. P. 1985, ApJ, 295, 422

Considère, S., \& Athanassoula, E. 1982, A\&A, 111, 28

Considère, S., \& Athanassoula, E. 1988, A\&AS, 76, 365

Crampton, D., \& Georgelin, Y. M. 1975, A\&A, 40, 317

Dame, T. M., Elmegreen, B. G., Cohen, R. S., \& Thaddeus, P. 1986, ApJ, 305, 892

Dame, T. M., \& Thaddeus, P. 1985, ApJ, 297, 751

Dambis, A. K., Mel'nik, A. M., \& Rastorguov, A. S. 1995, Astro. Lett., 21, 291

Danver, C. G. 1942, Ann. Obs. Lund., 10

Dehnen, W., \& Binney, J. J. 1998, MNRAS, 298, 387

Digel, S., Lyder, D. A., Philbrick, A. J., et al. 1996, ApJ, 458, 561

Digel, S., de Geus, E., \& Thaddeus, P. 1994, ApJ, 422, 92

Djorgovski, S., \& Sosin, C. 1989, ApJ, 341, L13

Downes, D., Wilson, T. L., Bieging, J., \& Wink, J. 1980, A\&AS, 40, 379

Elmegreen, B. G. 1990, in Dynamics of galaxies and their molecular cloud distribution (Kluwer, Dordrecht), ed. F. Combes, \& F. Casoli, Proc. IAU Symp., 146, 120

Elmegreen, D. M., \& Elmegreen, B. G. 1995, ApJ, 445, 591

Englmaier, P., \& Gerhard, O. 1999, MNRAS, 304, 512

Feinstein, A. 1994, in The formation of the MW, ed. E. J. Alfaro \& A. J. Delgado
Fich, M., \& Blitz, L. 1984, ApJ, 279, 125

Fich, M., Blitz, L., \& Stark, A. A. 1989, ApJ, 342, 272

Fich, M., Dahl, G. P., \& Treffers, R. R. 1990, AJ, 99, 622

Forbes, D. 1988, A\&AS, 77, 439

Georgelin, Y. M. 1975, Thesis, Université de Provence

Georgelin, Y. M., Amram, P., Georgelin, Y. P., et al. 1994, A\&AS, 108,513

Georgelin, Y. P., \& Georgelin, Y. M. 1971, A\&A, 12, 482

Georgelin, Y. M., \& Georgelin, Y. P. 1976, A\&A, 49, 57

Georgelin, Y. M., Russeil, D., Amram, P., et al. 2000, A\&A, 357, 308

Georgelin, Y. M., Russeil, D., Marcelin, M., et al. 1996, A\&AS, 308, 588

Gonzalez Delgado, R. M., \& Perez, E. 1997, ApJS, 108, 199

Griffith, M. R., Heflin, M., Conner, S., Burke, B., \& Langston, G. 1991, ApJS, 75, 801

Griffith, M. R., Langston, G., Heflin, M., et al. 1990, ApJS, 74, 129

Griffith, M. R., \& Wright, A. E. 1993, AJ, 105, 1666

Griffith, M. R., Wright, A. E., Burke, B. F., \& Ekers, R. D. 1995, ApJS, 97, 347

Guibert, J., Lequeux, J., \& Viallefond, F. 1978, A\&A, 68, 1

Gunn, J. E., Knapp, G. R., \& Tremaine, S. D. 1979, AJ, 84, 1181

Hayakawa, S., Matsumoto, T., Murakami, H., et al. 1981, A\&A, 100, 116

Heyer, M. H., \& Terebey, S. 1998, ApJ, 502, 265

Hodge, P. W., \& Kennicutt, R. C. 1983, AJ, 88, 296

Honma, M., \& Sofue, Y. 1996, PASJ, 48, L103

Hubble, E. 1926, ApJ, 64, 321

Humphreys, R. M. 1970, AJ, 75, 602

Humphreys, R. M. 1972, A\&A, 20, 29

Humphreys, R. M., \& Mac Elroy, D. B. 1984, ApJ, 284, 565

Jacq, T., Despois, D., \& Baudry, A. 1988, A\&A, 195, 93

Kaltcheva, N. T., \& Hilditch, R. W. 2000, MNRAS, 312, 753

Kennicutt, R. 1981, AJ, 86, 1847

Kennicutt, R. 1982a, AJ, 87, 255

Kennicutt, R. 1982b, ApJ, 253, 101

Kerr, F. J. 1957, AJ, 62, 93

King, I. 1989, in The Milky way as a galaxy, SAAS-FEE (Gilmore et al.), 63

Langston, G., Heflin, M., Conner, S., et al. 1990, ApJS, 72, 621

le Coarer, E., Amram, P., Boulesteix, J., et al. 1992, A\&A, 257, 389

Loktin, A. V., Matkin, N. V., \& Gerasimenko, T. P. 1994, Astron. Astrophys. Trans., 4, 153

Lòpez-Corredoira, M., Garzòn, F., Beckman, J. E., et al. 1999, AJ, 118,381

Lynga, G. 1987, Catalogue of Open Cluster Data (5th Ed.), Lund Observatory

Marcelin, M., Georgelin, Y. M., Amram, P., et al. 1995, in 3D optical Spectroscopic Method in Astronomy, ed. G. Comte \& M. Marcelin, ASP Conf. Ser., 71, 160

Merrifield, M. R. 1992, AJ, 103, 1552

Mezger, P. G., \& Henderson, A. P. 1967, ApJ, 147, 471

Miyamoto, M., Soma, M., \& Yoshizawa, M. 1993, AJ, 105, 2138

Nelder, J. A., \& Mead, R. 1965, Comp. J., 7, 308

Oey, M. S., \& Massey, P. 1994, ApJ, 425, 635

Olling, R. P., \& Merrifield, M. R. 1998, MNRAS, 297, 943

Ortiz, R., \& Lépine, J. R. D. 1993, A\&A, 279, 90

Perryman, M., Brown, A., Lebreton, Y., et al. 1998, A\&A, 331, 81

Persic, M., Salucci, P., \& Stel, F. 1996, MNRAS, 281, 27

Pont, F., Mayor, M., \& Burk, G. 1994, A\&A, 285, 415

Reid, M. J. 1993, ARA\&A, 31, 345

Reifenstein, E. C., Wilson, T. L., Burke, B. F., Mezger, P. G., \& Altenhoff, W. J. 1970, A\&A, 4, 357

Rickard, J. J. 1968, ApJ, 152, 1019 
Roberts, W. W. 1969, ApJ, 158, 23

Rohlfs, K., Chini, R., Wink, J. E., \& Bohme, R. 1986, A\&A, 158, 181

Rosado, M., Laval, A., le Coarer, E., et al. 1996, A\&A, 308, 58

Rozas, M., Zurita, A., Heller, C. H., \& Beckman, J. E. 1999, A\&AS, 135,145

Rozas, M., Zurita, A., \& Beckman, J. E. 2000, A\&A, 354, 823

Rubin, V. C., Burstein, D., Ford, W. K., \& Thonnard, N. 1985, ApJ, 289, 81

Ruprecht, J., Balazs, B., \& White, R. E. 1983, SvA, 27, 358

Russeil, D. 1997, A\&A, 319, 788

Russeil, D. 1998, Thesis, Université de Provence

Russeil, D., \& Castets, A. 2002, A\&A, submitted

Russell, W. S., \& Roberts, W. W. 1992, APJ, 398, 94

Sabas, S. 1997, Thesis, Université Paris VII

Schmidt-Kaler, Th. 1983, Landolt-Borstein, New Series, Group VI, Vol 2B, 14

Schraml, J., \& Mezger, P. G. 1969, ApJ, 156, 269

Seiden, P. E., \& Gerola, H. 1979, ApJ, 233, 56

Seigar, M. S., \& James, P. A. 1998, MNRAS, 299, 685
Sinha, S. P. 1978, A\&A, 69, 227

Sitnik, T. G., \& Mel'Nik, A. M. 1996, AstL, 22, 422

Sodroski, T. J. 1991, ApJ, 366, 95

Solomon, P. M., Rivolo, A. R., Barrett, J., \& Yahil, A. 1987, ApJ, 319, 730

Smart, R. L., Drimmel, R., \& Lattanzi, M. G. 1997, Proc. of the ESA Symp. "Hypparcos-Venice 97", 175

Vacca, W. D., Garmany, C. D., \& Shull, J. M. 1996, ApJ, 460, 914

Vallée, J. P. 1995, ApJ, 454, 119

Vorontsov-Vel'yaminov, B. A. 1987, ExtraGalactic astronomy (Harwood academic publishers)

Wilson, T. D., Barnes, T. G., Hawley, S. L., \& Jeffreys, W. H. 1991, ApJ, 378, 708

Wilson, T. L. 1972, A\&A, 19, 354

Wright, A. E., Griffith, M. R., Burke, B. F., \& Ekers, R. D. 1994, ApJS, 91,111

Yuan, C. 1969, ApJ, 158, 871

Zuckerman, B., \& Palmer, P. 1974, ARA\&A, 12, 279 